\title{
SYNCHRONIZATION AND NON-SMOOTH DYNAMICAL SYSTEMS
}

\author{
JAUME LLIBRE ${ }^{1}$, PAULO R. DA SILVA ${ }^{2}$ AND MARCO A. TEIXEIRA ${ }^{3}$
}

\begin{abstract}
In this article we establish an interaction between nonsmooth systems, geometric singular perturbation theory and synchronization phenomena. We find conditions for a non-smooth vector fields be locally synchronized. Moreover its regularization provide a singular perturbation problem with attracting critical manifold. We also state a result about the synchronization which occurs in the regularization of the fold-fold case. We restrict ourselves to the 3-dimensional systems $(\ell=3)$ and consider the case known as a T-singularity.
\end{abstract}

\section{INTRODUCTION}

The main goal of this paper is to establish an interaction between three important themes of the qualitative theory of non-smooth dynamical systems:

- synchronization phenomena,

- sliding vector fields (also known as Filippov systems) and

- singular perturbation.

Synchronization of dynamical systems has lately been an object of interest of many researchers from many areas as electrical and mechanical engineering, biology, and physics (see for instance [5]). The concept of synchronization used in this paper was inspired by Chow and Liu [4]. Let $\mathcal{U} \subseteq \mathbb{R}^{\ell}$ be an open set and $X: \mathcal{U} \rightarrow \mathbb{R}^{\ell}$ be a $C^{r}$ vector field, with $r \geq 1$, defining the dynamical system

$$
\dot{x}=X(x) .
$$

As usual $\varphi\left(t, x_{0}\right)$ denotes the solution of system (1) satisfying $\varphi\left(0, x_{0}\right)=x_{0}$. We say that system (1) is $\boldsymbol{M}$-synchronized if there exists a continuous map $G: \mathcal{U} \subseteq \mathbb{R}^{\ell} \rightarrow \mathbb{R}$ such that $M=G^{-1}(0)$ and $\lim _{t \rightarrow \infty} G\left(\varphi\left(t, x_{0}\right)\right)=0$, for any $x_{0} \in \mathcal{U}$. There are many mathematical methods to study synchronization in dynamical systems.

Example 1. If $p \in \mathbb{R}^{\ell}$ is an asymptotically stable equilibrium point of (1), then there exists $\mathcal{U} \subseteq \mathbb{R}^{\ell}$ such that for any $q \in \mathcal{U}$ we have that $\lim _{t \rightarrow \infty} \varphi(t, q)=$ $p$. Thus taking $G(x)=\|x-p\|$ we have that (1) is $M$-synchronized at $\mathcal{U}$ with

1991 Mathematics Subject Classification. Primary 34C20, 34C26, 34D15, 34H05.

Key words and phrases. Regularization, vector field, constrained system, singular perturbation, non-smooth vector field, sliding vector field, synchronization. 
$M=G^{-1}(0)=\{p\}$. In particular if we have a Liapunov function $V$ then the synchronization occurs. However, sometimes it is difficult to prove that $\dot{V}<0$. Rodrigues [1] presented a general version of the invariance principle in which the derivative of the Liapunov function is not required.

Example 2. If $M \subseteq \mathbb{R}^{2}$ is a stable or semi-stable limit cycle of $X$ then following our approach it is possible to get a $M$-synchronization phenomenon. See for instance [13]. In particular the averaging method offers an algorithm to detect existence of limit cycles.

Example 3. We point out that if the smoothness conditions are dropped synchronization phenomenon can also occur. Consider the piecewise smooth vector field on the plane given by

$$
X(x, y)=\left\{\begin{array}{lll}
X^{+}(x, y)=(-y,-x) & \text { if } & y>0 \\
X^{-}(x, y)=(-1,1) & \text { if } & y<0
\end{array}\right.
$$

The system is $M$-synchronized with $M=G^{-1}(0)$ and

$$
G(x, y)= \begin{cases}y+x & \text { if } x \leq 0 \\ y & \text { if } x>0\end{cases}
$$

See figure 1.

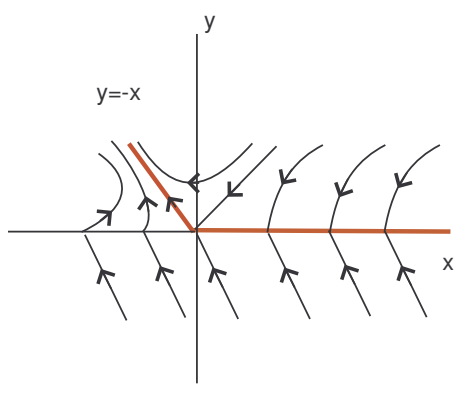

Figure 1. $M$-Synchronized discontinuous vector field. The bold line is the set $M$.

Now we illustrate how the singular perturbation techniques can be useful in the investigation of synchronization problems.

Example 4. Consider the two parameter family of vector fields $X_{\varepsilon, \psi}$ on the plane given by

$$
X_{\varepsilon, \psi}(x, y)=(x y+\varepsilon(\sin \psi+f(x, y, \varepsilon)), x y+\varepsilon(\cos \psi+g(x, y, \varepsilon))
$$

where $\psi \in\left(\frac{\pi}{4}, \frac{\pi}{2}\right), \varepsilon \geq 0, f(0,0,0)=g(0,0,0)=0$ and with $f, g$ of class $C^{\infty}$. Observe that the singular set of $X_{0,0}$ is represented by the equation $x y=0$. The study of the phase portrait for $\varepsilon>0$ but small can be made by considering center manifolds along the curve of zeroes. We refer [7] for the details of the technique called "family blow up" that will be used here. There exists a regular trajectory $\gamma$ which divides the plane in two open sets having $\gamma$ like a common boundary. Moreover, on each one of these open 
sets the system is locally synchronized. The process produces a limiting surface composed by the disc $D(0,1)=\left\{x \in \mathbb{R}^{2} ;|x| \leq 1\right\}$ and by the plane $\mathbb{R}^{2} \backslash\{(0,0)\}$. The dynamics on $D(0,1)$ is the dynamics of the system

$$
\dot{\bar{x}}=\overline{x y}+\sin (\psi), \quad \dot{\bar{y}}=\overline{x y}+\cos (\psi) .
$$

We provide the global phase portrait of this system in the Poincaré ball (i.e. in the compactification of $\mathbb{R}^{2}$ with the sphere $\mathbb{S}^{1}$ of the infinity). The phase portrait is drawn by the programme $\mathrm{P} 4$ and it is illustrated in figure 2 , for details see [6]. In polar coordinates $x=r \cos \theta, y=r \sin \theta, X_{\varepsilon, \psi}$ takes the form

$$
\begin{aligned}
& \dot{r}=r^{2}\left(\cos ^{2} \theta \sin \theta+\sin ^{2} \theta \cos \theta\right)+\varepsilon(\cos \theta(\sin \psi+f)+\sin \theta(\cos \psi+g)), \\
& \dot{\theta}=r\left(\cos ^{2} \theta \sin \theta-\sin ^{2} \theta \cos \theta\right)+\frac{\varepsilon}{r}(-\sin \theta(\sin \psi+f)+\cos \theta(\cos \psi+g)) .
\end{aligned}
$$

Moreover we consider $\varepsilon=0$ and define $Y_{0, \psi}=\frac{X_{0, \psi}}{r}$. The rays $\theta=0, \pi / 2, \pi$ and $3 \pi / 2$ are composed by equilibrium points and the rays $\theta=\pi / 4$ and $\theta=5 \pi / 4$ are invariant by the flow. Thus the phase portrait of the limiting system is illustrated on Figure 2. Using the geometric singular perturbation theory we have that the phase portrait of $X_{\varepsilon, \psi}$, for $\psi \in\left(\frac{\pi}{4}, \frac{\pi}{2}\right)$ and $\varepsilon>0$ small, is illustrated in figure 2.
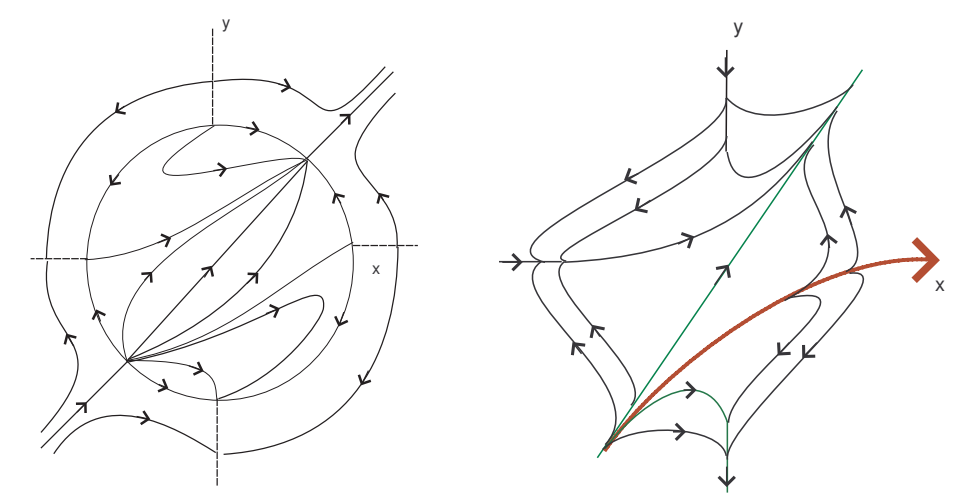

FIGURE 2. Phase portrait of the limiting system and the phase portrait of $X_{\varepsilon, \psi}$, for $\psi \in\left(\frac{\pi}{4}, \frac{\pi}{2}\right)$ and $\varepsilon>0$ small. The bold line divides the plane in two open sets. On each one of the open sets the system is locally synchronized.

It is worth mentioning that there are some results on synchronization in the framework of invariant manifold theory. Normal hyperbolicity and its persistence can be applied to get general results on synchronization. In [4] it is proved that if $M$ is an invariant set and all Lyapunov exponents on $M$ are negative, then the system is $M$-synchronized.

The paper is organized as follows. In Section 2 we present basic facts and definitions about non-smooth dynamical systems. In Section 3 we state our main results. In Section 4 we prove the main results. 


\section{NON-SMOOTH DYNAMICAL SYSTEMS}

Let $\mathcal{U} \subseteq \mathbb{R}^{\ell}$ be an open subset with $0 \in \mathcal{U}$. A non-smooth dynamical system can be considered as a pair of differential systems in the following way. Consider a $C^{r}$ map $H: \mathcal{U} \subseteq \mathbb{R}^{\ell} \rightarrow \mathbb{R}$ satisfying that $H(0)=0$ and $\nabla H(0) \neq 0$. Thus we can assume that $\Sigma=\{p \in \mathcal{U} ; H(p)=0\}$ is a smooth submanifold through 0 . Denote $\Sigma_{+}=\{p \in \mathcal{U} ; H(p)>0\}$ and $\Sigma_{-}=\{p \in \mathcal{U} ; H(p)<0\}$.

Denote $\mathcal{X}^{r}, r \geq 1$, the set of $\mathcal{C}^{r}$ vector fields defined on $\mathcal{U}$. The topology on $\mathcal{X}^{r}$ is the usual $\mathcal{C}^{r}$ topology. Denote $\Omega^{r}(\mathcal{U}, H)$ the set of vector fields $Z=\left(X^{+}, X^{-}\right)$where $X^{+}$and $X^{-}$are defined on $\mathcal{U}$. We consider the product topology on $\Omega^{r}(\mathcal{U}, H)$. The vector fields $\left(X^{+}\right)$and $\left(X^{-}\right)$define flows $\varphi^{+}(t, p)$ and $\varphi^{-}(t, p)$ which provide $C^{r}$-foliations on $\mathcal{U} \cap \Sigma_{+}$and on $\mathcal{U} \cap \Sigma_{-}$, respectively.

There is a distinguished region $\Sigma^{S} \subseteq \Sigma$ characterized by the following property: when a trajectory $\gamma(t)$ of $\left(X^{+}, X^{-}\right) \in \Omega^{r}(\mathcal{U}, H)$ meets $\Sigma$ at $p \in \Sigma^{S}$ it slides on $\Sigma$ for positive time. The study of this flow is our main concern.

Let $\mathcal{U} \subseteq \mathbb{R}^{\ell}$ be an open set with $0 \in \mathcal{U}$ and $\left(X^{+}, X^{-}\right) \in \Omega^{r}(\mathcal{U}, H)$ with discontinuity set $\Sigma$. The Filippov convention ( see [9]) is the following: the sewing region is $\Sigma_{1}=\left\{p \in \Sigma: X^{+} H(p) \cdot X^{-} H(p)>0\right\}$, the escaping region is $\Sigma_{2}=\left\{p \in \Sigma: X^{+} H(p)>0, X^{-} H(p)<0\right\}$, and the sliding region is $\Sigma_{3}=\left\{p \in \Sigma: X^{+} H(p)<0, X^{-} H(p)>0\right\}$.

On $\Sigma^{S}=\Sigma_{2} \cup \Sigma_{3}$ the flow slides on $\Sigma$; the flow follows a well defined vector field $X^{S}$ called sliding vector field. It is tangent to $\Sigma$ and defined at $q \in \Sigma^{S}$ by $X^{S}(q)=m-q$ with $m$ being the point where the segment joining $q+X^{+}(q)$ and $q+X^{-}(q)$ is tangent to $\Sigma$ ( see $[3,10,11,12]$ for more details and related topics).

An approximation of the discontinuous vector field $\left(X^{+}, X^{-}\right) \in \Omega^{r}(\mathcal{U}, H)$ by an one-parameter family of continuous vector fields will be called a regularization of $\left(X^{+}, X^{-}\right)$. In the work [14] Sotomayor and Teixeira introduced the regularization process. A $C^{\infty}$ function $\varphi: \mathbb{R} \longrightarrow \mathbb{R}$ is a transition function if $\varphi(x)=-1$ for $x \leq-1, \varphi(x)=1$ for $x \geq 1$ and $\varphi^{\prime}(x)>0$ if $x \in(-1,1)$. Consider a $C^{r}$ map $H: \mathcal{U} \subseteq \mathbb{R}^{\ell} \rightarrow \mathbb{R}$ satisfying that $H(0)=0$ and $\nabla H(0) \neq(0,0)$. The $\varphi$-regularization of $X=\left(X^{+}, X^{-}\right) \in \Omega^{r}(\mathcal{U}, H)$ is the 1-parameter family $X_{\varepsilon} \in C^{r}$ given by

$$
X_{\varepsilon}(q)=\left(\frac{1}{2}+\frac{\varphi_{\varepsilon}(H(q))}{2}\right) X^{+}(q)+\left(\frac{1}{2}-\frac{\varphi_{\varepsilon}(H(q))}{2}\right) X^{-}(q),
$$

where $\varphi_{\varepsilon}(x)=\varphi(x / \varepsilon)$, for $\varepsilon>0$.

2.1. Singular perturbations. Geometric singular perturbation theory (GSPtheory) is an important tool in the field of continuous dynamical systems. Needless to say that in this area very good surveys are available (refer to $[7,8])$. 
Let $w=(x, y) \in \mathbb{R}^{n+m}$ and $f, g$ be smooth functions. We deal with equations that may be written in the form

$$
x^{\prime}=f(x, y, \varepsilon), \quad y^{\prime}=\varepsilon g(x, y, \varepsilon), \quad x=x(\tau), \quad y=y(\tau) .
$$

The main trick in the GSP-theory consists in considering the family (2) in addition with the family (3) obtained after the time rescaling $t=\varepsilon \tau$.

$$
\varepsilon \dot{x}=f(x, y, \varepsilon), \quad \dot{y}=g(x, y, \varepsilon), \quad x=x(t), \quad y=y(t) .
$$

Equation (2) is called the fast system and (3) the slow system. Observe that for $\varepsilon>0$ the phase portrait of fast and slow systems coincide. For $\varepsilon=0$, let $\mathcal{M}$ be the set of all singular points of (2). We call $\mathcal{M}$ the slow manifold of the singular perturbation problem and it is important to notice that equation (3) defines a dynamical system, on $\mathcal{M}$, called the reduced problem.

Combining results on the dynamics of these two limiting problems one obtains information on the dynamics for small values of $\varepsilon$. In fact, such techniques can be exploited to formally construct approximated solutions, on pieces of curves which satisfy some limiting version of the original equation as $\varepsilon$ goes to zero.

2.2. Connection between the regularization process and singular perturbation problems. A singular perturbation problem is a study of the phase portrait of a differential system, $\dot{x}=X_{\varepsilon}(x)$ with $x \in \mathbb{R}^{\ell}$ and $\varepsilon \sim 0$, near a continuum of zeroes of $X_{0}(x)$. This set can be a differentiable manifold, an algebraic variety or a general stratified set. Fenichel [8] studied the existence of centermanifolds and the reduction principle to central behavior in the neighborhood of compact pieces of normally hyperbolic regular manifolds of zeroes. Roughly speaking, if $\ell=2$ and the center manifold is attracting in the normal direction then for $\varepsilon \sim 0$, there exists a curve $\gamma$ which is near the center manifold and which is a global attractor of $X_{\varepsilon}$. Usually we say that when there exists a graphic with the attracting property the system is synchronized. Here we propose to use singular perturbation as a framework to study synchronization.

In order to illustrate our techniques we present an example of a nonsmooth dynamical system and its regularized. Observe that by means of a blow up procedure it appears naturally a singular perturbation problem. Thus the regularized vector field will be synchronized for small values of the the parameter and one gets informations on the original vector field.

Example 5. Consider the vector fields $X^{-}=(x+1,-y+1)$ and $X^{+}=$ $(0,1)$ defined on $\mathbb{R}^{2}$. Suppose that $X$ is a non-smooth vector field which is equal to $X^{-}$if $y>x$, and it is equal to $X^{+}$if $y<x$. On the line $\Sigma=\{(x, x) ; x \in \mathbb{R}\}$ we assume that $X$ is multi-valuated. Observe that the points $(x, x) \in \Sigma$ with $x<0$ are sewing points, and the points $(x, x) \in \Sigma$ with $x>0$ are sliding points. The phase portrait of $X$ is illustrated in figure 3 . 


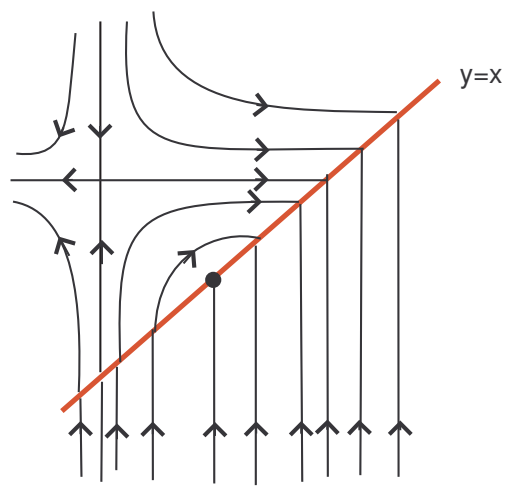

Figure 3. Non-smooth vector field $X=\left(X^{+}, X^{-}\right)$with $X^{-}=(x+1,-y+1)$ and $X^{+}=(0,1)$.

To simplify the regularization process we start with a rotation of $\pi / 4$. Thus we get the pair of vector fields

$$
Y^{+}=\frac{\sqrt{2}}{2}(-1,1), \quad x>0 ; \quad Y^{-}=\frac{\sqrt{2}}{2}(x+y, x-y+2), \quad x<0 .
$$

Consider $\varphi: \mathbb{R} \rightarrow(-\pi / 2, \pi / 2)$ given by $\varphi(s)=\frac{2}{\pi} \arctan (s)$. It is a $C^{\omega}$ function satisfying $\varphi^{\prime}(s)>0$ for $s \in \mathbb{R}$ and $\lim _{s \rightarrow \pm \infty}= \pm 1$. Around $\widetilde{\Sigma}=\{x=0\}$ we apply the regularization $Y_{\varepsilon}=\frac{Y^{+}+Y^{-}}{2}+\varphi\left(\frac{x}{\varepsilon}\right) \frac{Y^{+}-Y^{-}}{2}$. We transform this system into a singular perturbation problem by considering $x=r \cos \theta, \varepsilon=r \sin \theta$, with $r \geq 0$ and $\theta \in[0, \pi]$. We get

$$
\begin{aligned}
& r \dot{\theta}=\frac{\sqrt{2}}{4} \sin \theta(1-r \cos \theta-y+\varphi(\cot \theta)(1+r \cos \theta+y)), \\
& \dot{y}=\frac{\sqrt{2}}{4}(3+r \cos \theta-y+\varphi(\cot \theta)(-1-r \cos \theta+y)),
\end{aligned}
$$

where $r \in \mathbb{R}^{+}, \theta \in[0, \pi], y \in \mathbb{R}$. Observe that the mapping $\lambda(\theta)=\varphi(\cot \theta)$ can be extended to a continuous decreasing function connecting $(\theta, \lambda)=$ $(0,1)$ and $(\theta, \lambda)=(\pi,-1)$. Putting $r=0$ in the first equation we get the slow manifold $\varphi(\cot \theta)=\frac{y-1}{y+1}$, which connects the points $(\theta, y)=(0,0)$ and $(\theta, y)=(\pi, \infty)$. The slow flow, i.e, the flow determined by the second equation on the the slow manifold is given by

$$
\dot{y}=(3-y)+\frac{y-1}{y+1}(y-1)>0 .
$$

The fast flow, i.e, the flow determined by the first equation after the time re-scale, is given by $\theta^{\prime}=\frac{\sqrt{2}}{4} \sin \theta \cdot(1-y+\varphi(\cot \theta)(1+y))$.

Observe that in our approach we define a geometric object composed by the union of $\widetilde{\Sigma}_{+}=\{x>0\}, \widetilde{\Sigma}_{-}=\{x<0\}$ and $\widetilde{\Sigma}_{0}=\{(\theta, y) ; \theta \in$ $(0, \pi),(0, y) \in \widetilde{\Sigma}\}$. Thus we use the following rules for defining the orbits: if a point of $\widetilde{\Sigma}_{+} \cup \widetilde{\Sigma}_{-}$moving on an orbit of $Y^{\sigma}, \sigma=+,-$, falls onto the sewing region then it crosses $\widetilde{\Sigma}$ through a fast orbit; if it falls onto the sliding then 


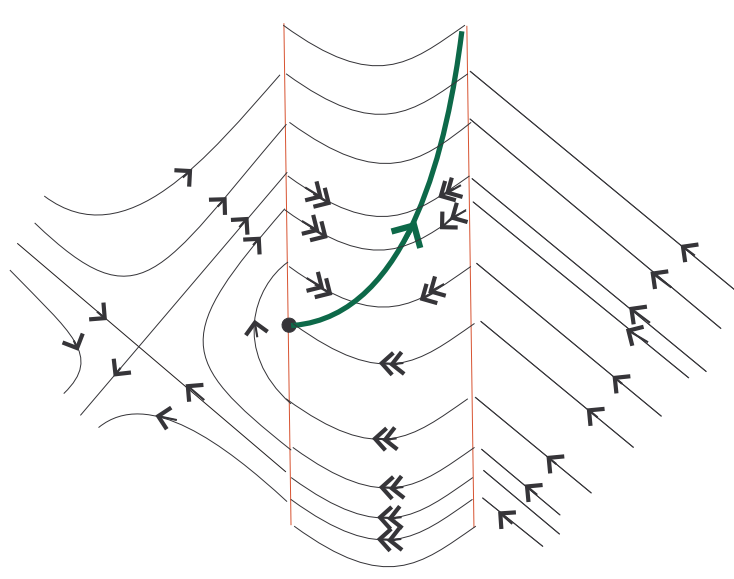

Figure 4. Phase portrait on the singular set $r=0$ of $Y=\left(Y^{+}, Y^{-}\right)$. The double arrow is related with the fast system and the simple arrow is related with the slow system. The bold line is the slow manifold $\mathcal{S}_{0}$. For $r \downarrow 0$ there exists a curve $\mathcal{S}_{\varepsilon}$ which approaches $\mathcal{S}_{0}$ according the Hausdorff distance and that synchronizes the regularized vector field.

it follows firstly a fast orbit over the slow manifold and then it follows a slow orbit. See figure 4.

\section{Statement of the MAin RESults}

Our main results link non-smooth systems and singular perturbation problems. First of all, for completeness, we present here a first theorem, which proof can be found in [11].

Theorem A. Let $\mathcal{U} \subseteq \mathbb{R}^{2}$ be an open set. Consider a $C^{r}$ map $H: \mathcal{U} \subseteq$ $\mathbb{R}^{2} \rightarrow \mathbb{R}$ satisfying that $H(0)=0$ and $\nabla H(0) \neq 0$ and $X \in \Omega^{r}(\mathcal{U}, H)$. If $p \in \Sigma \cap \mathcal{U}$ then there exist a local coordinate system $(x, y)$ with $p=(0, y(p))$ and a singular perturbation problem $\theta^{\prime}=\alpha(r, \theta, y), y^{\prime}=r \beta(r, \theta, y)$, such that

- $\Sigma^{S}$ is homeomorphic to the slow manifold $\{\alpha(0, \theta, y)=0\}$,

- $X^{\mathcal{S}}$ is topologically equivalent to the slow flow

$$
\left(0, y^{\prime}\right)=(\alpha(0, \theta, y), \beta(0, \theta, y)),
$$

- If $p \in \Sigma^{1}$ then the flow of $X$ on $p$ is locally topologically equivalent to the fast flow

$$
\left(\theta^{\prime}, y^{\prime}\right)=(\alpha(0, \theta, y), 0) .
$$

Let $X=\left(X^{+}, X^{-}\right) \in \Omega^{r}(\mathcal{U}, H)$ be a discontinuous vector field defined on the open set $\mathcal{U} \subseteq \mathbb{R}^{\ell}$. As in the smooth case we denote $\varphi(t, p)$ the trajectory of $X$ which satisfies $\varphi(0, p)=p$. Here, the concept of solution follows the Filippov rules. More precisely if $q=\varphi\left(t^{*}, p\right) \in \Sigma^{S}$ then the flow over $q$ will 
be the flow determined by the sliding vector field. The basin of attraction of $\Sigma^{S}$ is the set $\mathcal{A}_{\Sigma}=\left\{p \in \mathbb{R}^{\ell} ; \varphi(t, p) \in \Sigma^{S}\right.$ for some $\left.t>0\right\}$.

Theorem B. $X \in \Omega^{r}(\mathcal{U}, H)$ is $M$-synchronized at $\mathcal{A}_{\Sigma}$ with $M=\Sigma=$ $H^{-1}(0)$.

Our third result is about the synchronization which occurs in the regularization of the fold-fold case. We restrict ourselves to the 3-dimensional systems $(\ell=3)$ and consider the case known as a T-singularity.

We say that $q \in \Sigma$ is a codimension 0 singular point of $Z=\left(X^{+}, X^{-}\right)$of the kind fold-fold if

$$
X^{+}(H)(q)=X^{-}(H)(q)=0, \quad\left(X^{+}\right)^{2}(H)(q) \neq 0, \quad\left(X^{-}\right)^{2}(H)(q) \neq 0
$$

and the curves $\sigma_{X^{+}}, \sigma_{X^{-}}$, along which $X^{+}$and $X^{-}$has quadratic contact with $\Sigma$, are transverse at $q$. Besides, when $\left(X^{+}\right)^{2}(H)(q)<0$ and $\left(X^{-}\right)^{2}(H)(q)>0$ we say that $q$ is a $\boldsymbol{T}$-singularity.

Theorem C (Semi-linear case). Assume $H(x, y, z)=z, X_{+}=\left(k_{1}, a, x\right)$ and $X_{-}=\left(b, k_{2}, y\right)$. If $k_{1}<0, k_{2}>0, a+b<0, a<b$ and $a b<k_{1} k_{2}$, then $(0,0)$ is a T-singularity. Moreover the sliding vector field is synchronized with respect to $\mathcal{U}=\{x<0, y>0\}$ and $M=\{(0,0)\}$.

Our fourth result says that every structure of the slow system which persists under regular perturbation also persists under singular perturbation. More specifically, consider the differential system

$$
\dot{x}=f(x, y, \varepsilon), \quad \dot{y}=\varepsilon g(x, y, \varepsilon)
$$

with $(x, y) \in \mathbb{R}^{n+m}$ and the slow manifold $\mathcal{S}$ given implicitly by $f(x, y, 0)=$ 0 . We say that $p \in \mathcal{S}$ is normally hyperbolic if the real parts of the eigenvalues of $D_{1} f(p, 0)$ are nonzero. We assume that, for every normally hyperbolic $p \in \mathcal{S}, D_{1} f(p, 0)$ has $k^{s}$ eigenvalues with negative real part.

Theorem D. Let $q \in \mathcal{S}$ be a hyperbolic equilibrium point of the slow flow with $j^{s}$-dimensional local stable manifold $W^{s}$. Then there exists an $\varepsilon$ continuous family of equilibrium points $q_{\varepsilon}$ such that $q_{0}=q$ and $q_{\varepsilon}$ has a $\left(k^{s}+j^{s}\right)$-dimensional local stable manifold $W_{\varepsilon}^{s}$. In particular, if $k^{s}+j^{s}=$ $n+m$, for small $\varepsilon>0$ the systems is synchronized with respect to $\mathbb{R}^{n+m}$ and $M=\left\{q_{\varepsilon}\right\}$.

\section{Proof of the MAin Results}

Proof of Theorem A. Denote $X_{+}=\left(f_{+}, g_{+}\right)$and $X_{-}=\left(f_{-}, g_{-}\right)$. First of all we apply the regularization process between $X_{+}$and $X_{-}$. Next we consider the polar blow up coordinates given by $x=r \cos \theta$ and $\varepsilon=r \sin \theta$, with $r \geq 0$ and $\theta \in[0, \pi]$. Using these coordinates the parameter value $\varepsilon=0$ is represented by $r=0$. We refer [11] for the details. 
Proof of Theorem B. In fact, for any $(x, y) \in \mathcal{A}_{\Sigma}$ there exists $\tau=\tau(x, y)>0$ such that $\varphi(t, x, y) \in \Sigma^{S}=\{H=0\}$, for any $t>\tau$. Taking $G=H$ we have

$$
\lim _{t \rightarrow \infty} G(\varphi(t, x, y))=\lim _{t \rightarrow \infty} H(\varphi(t, x, y))=\lim _{t \rightarrow \infty} 0=0 .
$$

Proof of Theorem C. As $H(x, y, z)=z, X_{+}=\left(k_{1}, a, x\right)$ and $X_{-}=\left(b, k_{2}, y\right)$, $(0,0,0)$ is a fold-fold singularity. The $\mathrm{SP}-$ problem in the blowing up locus is

$$
\begin{aligned}
\dot{x} & =\left(k_{1}+b\right) / 2+\varphi(\cot \theta)\left(k_{1}-b\right) / 2, \\
\dot{y} & =\left(a+k_{2}\right) / 2+\varphi(\cot \theta)\left(a-k_{2}\right) / 2, \\
r \dot{\theta} & =-\sin \theta((x+y) / 2+\varphi(\cot \theta)(x-y) / 2) .
\end{aligned}
$$

The slow manifold is the surface

$$
\left\{(x, y, \theta) \in \mathbb{R}^{2} \times(0, \pi):(x+y) / 2+\varphi(\cot \theta)(x-y) / 2=0\right\} .
$$

The graphic of this surface has a helicoidal shape and its intersections with the planes $\theta=$ constant are straight lines. For $\theta=0$ the line is $x=0$ and for $\theta=\pi$ the line is $y=0$.

The regularization $X_{\varepsilon}$ is given by

$$
\begin{gathered}
X_{\varepsilon}=\left(\frac{1}{2}+\frac{\varphi\left(\frac{z}{\varepsilon}\right)}{2}\right) X_{+}+\left(\frac{1}{2}-\frac{\varphi\left(\frac{z}{\varepsilon}\right)}{2}\right) X_{-}= \\
\left(\frac{k_{1}+b}{2}+\varphi\left(\frac{z}{\varepsilon}\right) \frac{k_{1}-b}{2}, \frac{a+k_{2}}{2}+\varphi\left(\frac{z}{\varepsilon}\right) \frac{a-k_{2}}{2}, \frac{x+y}{2}+\varphi\left(\frac{z}{\varepsilon}\right) \frac{x-y}{2}\right) .
\end{gathered}
$$

Performing the blow up $z=r \cos \theta, \varepsilon=r \sin \theta$, where $r \geq 0$ and $\theta \in[0, \pi]$ we get equation (4). Considering $r=0$ in the third line of the equation (4) we get the slow manifold. Observe that for each fixed $\theta \in[0, \pi]$ the points $(\theta, x, y)$ satisfying $\varphi(\cot \theta)=-\frac{x+y}{x-y}$ are on a straight line. Since $\varphi(\cot 0)=1$ and $\varphi(\cot \pi)=-1$ for $\theta=0$ the line is $x=0$ and for $\theta=\pi$ the line is $y=0$. It concludes the proof of (a). In order to prove (b) we determine the slow flow. It is given by

$$
\left(\begin{array}{c}
\dot{x} \\
\dot{y}
\end{array}\right)=\frac{1}{x-y}\left(\begin{array}{cc}
b & -k_{1} \\
k_{2} & -a
\end{array}\right)\left(\begin{array}{l}
x \\
y
\end{array}\right)=\frac{1}{y-x}\left(\begin{array}{cc}
-b & k_{1} \\
-k_{2} & a
\end{array}\right)\left(\begin{array}{l}
x \\
y
\end{array}\right) \text {. }
$$

Since $y-x>0$ in the sliding region $(x<0$ and $y>0)$ it is enough to compute the eigenvalues of the above matrix.

Since $k_{1}<0, k_{2}>0, a-b<0$, and $a b<k_{1} k_{2}$ the eigenvalues

$$
\lambda_{ \pm}=\frac{-(b-a) \pm \sqrt{(b-a)^{2}-4\left(k_{1} k_{2}-a b\right)}}{2}=\frac{a-b \pm \sqrt{(b+a)^{2}-4 k_{1} k_{2}}}{2}
$$

are negative. In fact, $-4 k_{1} k_{2}>0$ and then $(b+a)^{2}-4 k_{1} k_{2}>0$. It implies that the eigenvalues are real. Besides,

$$
a b<k_{1} k_{2} \Rightarrow(b+a)^{2}-4 k_{1} k_{2}<(b-a)^{2} .
$$


Since $(a-b)<0$ and $\sqrt{(b+a)^{2}-4 k_{1} k_{2}}<(b-a)=|a-b|$ the eigenvalues are negative. The corresponding eigenvectors

$$
\omega_{ \pm}=\left(\frac{2 k_{1}}{a+b \pm \sqrt{(a+b)^{2}-4 k_{1} k_{2}}}, 1\right)
$$

are located in the sewing and in the sliding regions respectively. Since $\left|\lambda_{-}\right|>\left|\lambda_{+}\right|$we have that all trajectories (except those on the straight line corresponding to $\omega_{-}$) tend to the origin tangentially to the straight line generated by $\omega_{+}$.

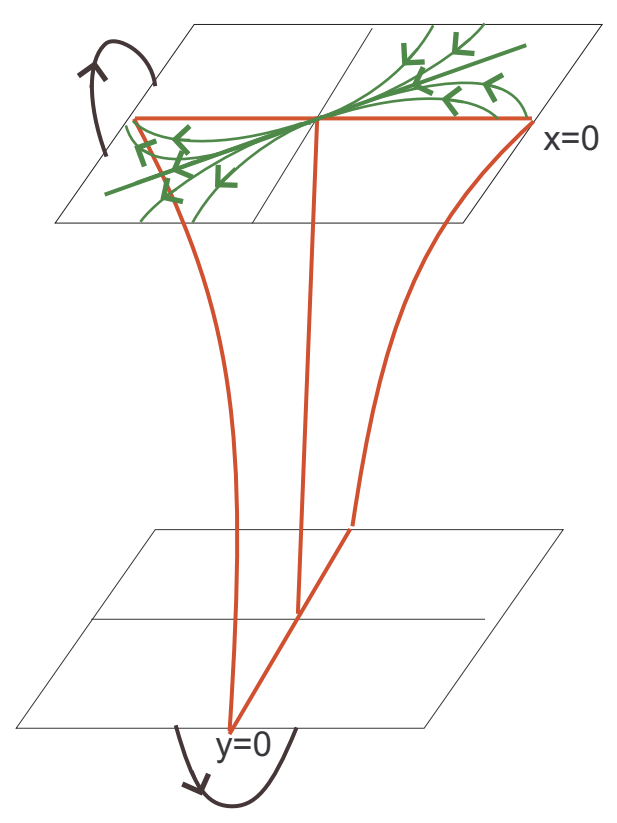

Figure 5. T-singularity.

Proof of Theorem D. Consider the fast system supplemented by the trivial equation $\varepsilon^{\prime}=0$, i.e.

$$
x^{\prime}=\varepsilon f(x, y, \varepsilon), \quad y^{\prime}=g(x, y, \varepsilon), \quad \varepsilon^{\prime}=0 .
$$

Let $G(x, y, \varepsilon):=(\varepsilon f(x, y, \varepsilon), g(x, y, \varepsilon), 0)$ be the vector field defined by the system (5). Assume that the linearization of $G$ at points $(x, y, 0)$, such that $(x, y) \in \mathcal{S}$, has $k^{s}$ eigenvalues with negative real part and $k^{u}$ eigenvalues with positive real part. The corresponding stable and unstable eigenspaces have dimensions $k^{s}$ and $k^{u}$, respectively. Now we are in position to use the Fenichel Lemma (see [8] for details). 
Lemma 1 (Fenichel Lemma). Let $\mathcal{N}$ be a $j$-dimensional compact normally hyperbolic invariant manifold of the reduced problem with a $j+j^{s}$ dimensional local stable manifold $W^{s}$ and $a\left(j+j^{u}\right)$-dimensional local unstable manifold $W^{u}$. Then there exists $\varepsilon_{1}>0$ for which the following statements hold.

(1) There exists a $C^{r-1}$ family of manifolds $\left\{\mathcal{N}_{\varepsilon}: \varepsilon \in\left(-\varepsilon_{1}, \varepsilon_{1}\right)\right\}$ such that $\mathcal{N}_{0}=\mathcal{N}$ and $\mathcal{N}_{\varepsilon}$ is a normal hyperbolic invariant manifold.

(2) There are $C^{r-1}$ families of $\left(j+j^{s}+k^{s}\right)$-dimensional and $\left(j+j^{u}+k^{u}\right)$ dimensional manifolds $\left\{\mathcal{N}_{\varepsilon}^{s}: \varepsilon \in\left(-\varepsilon_{1}, \varepsilon_{1}\right)\right\}$ and $\left\{\mathcal{N}_{\varepsilon}^{u}: \varepsilon \in\left(-\varepsilon_{1}, \varepsilon_{1}\right)\right\}$ such that for $\varepsilon>0$ the manifolds $\mathcal{N}_{\varepsilon}^{s}$ and $\mathcal{N}_{\varepsilon}^{u}$ are local stable and unstable manifolds of $\mathcal{N}_{\varepsilon}$.

Next example illustrates the application of Theorem D.

Example 6. Consider the one-parameter family of constrained systems in $\mathbb{R}^{2}$

$$
\dot{x}=x, \quad(x+y-\lambda) \dot{y}=y, \quad \lambda \in \mathbb{R} .
$$

Applying the change of variables $x_{1}=x, y_{1}=y$ and $\varepsilon=x+y-\lambda$, we get the following singular perturbation problem $\dot{x}=x, \varepsilon \dot{y}=y$. For $\varepsilon=0$ we have the reduced problem $\dot{x}=x, 0=y$. The time rescaling $\tau=t / \varepsilon$ produces $x^{\prime}=\varepsilon x, y^{\prime}=y$, and so for $\varepsilon=0$ we have the fast system $x^{\prime}=0, y^{\prime}=y$. The phase portraits of the reduced and of the fast systems are exhibited in Figure 6.
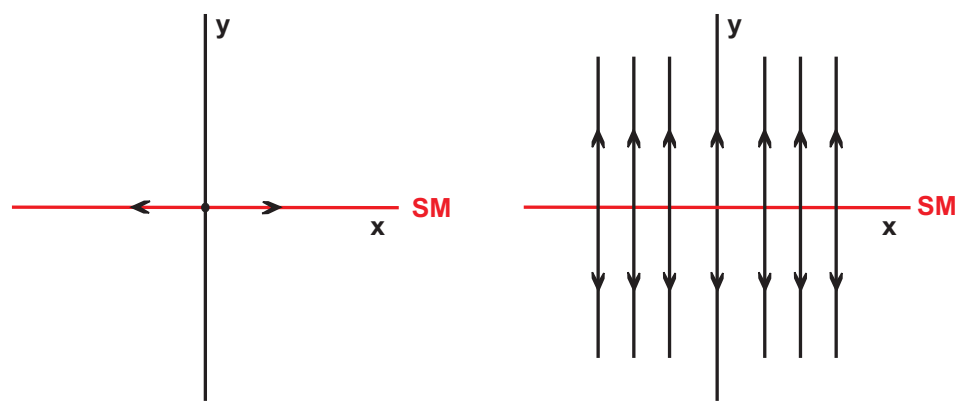

Figure 6. Phase portraits of reduced and fast of Example.

Figure 7 illustrates the phase portrait of the singular perturbation problem. The points of the form $(\lambda, 0, \lambda)$ are impasse-normally-hyperbolic points and correspond to the slow manifold of the singular perturbation problem.

Around $(0,0,0)$ Theorem D assures the existence of the curve $\gamma(\lambda)=$ $(0,0,-\lambda)$ and such that, for each fixed $\lambda,(0,0,-\lambda)$ is an equilibrium point for the adjoint vector field $F^{*}(x, y, \lambda)=A^{*} F(x, y, \lambda)=\left(x^{2}+x y-\lambda x, y\right)$. Moreover, each point $(0,0,-\lambda)$ with $\lambda<0$, possesses an 1-dimensional local stable manifold $P_{\lambda}^{s}$ and an 1-dimensional local unstable manifold $P_{\lambda}^{u}$. Also, for each $(0,0,-\lambda)$ with $\lambda>0$, there exists a 2-dimensional local unstable manifold $P_{\lambda}^{u}$. 

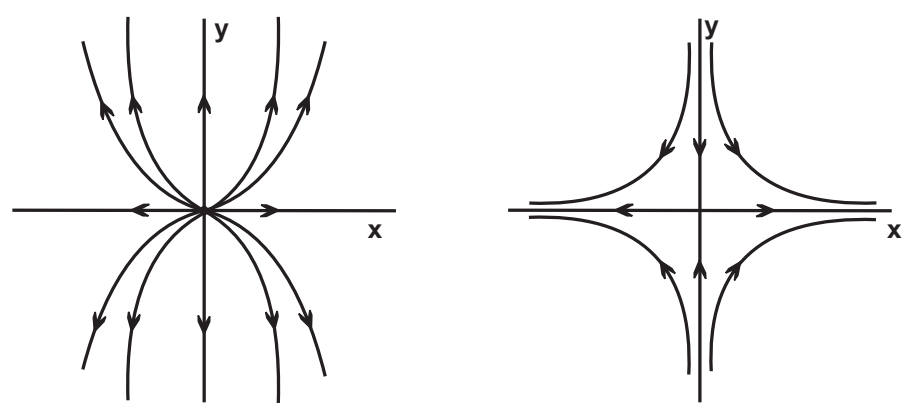

Figure 7. Phase portraits for small $\varepsilon>0$ and $\varepsilon<0$.

\section{REgUlARIZATION AND SYNCHRONIZATION}

First of all we introduce the concept of $\delta$-synchronization.

$$
x^{\prime}=f(x, y), \quad y^{\prime}=g(x, y) .
$$

As usual $\varphi\left(t, x_{0}, y_{0}\right)=\left(x\left(t, x_{0}, y_{0}\right), y\left(t, x_{0}, y_{0}\right)\right)$ denotes the solution of system $(7)$ satisfying $\varphi\left(0, x_{0}, y_{0}\right)=\left(x_{0}, y_{0}\right)$.

Consider $\delta>0$. We say that system (7) is $\delta$-synchronized for $t \in\left[t_{1}, t_{2}\right]$ and $(x, y) \in \mathcal{A} \subseteq \mathbb{R}^{2}$ if there exists a continuous map $G: \mathbb{R}^{2} \rightarrow \mathbb{R}$ such that $G^{-1}(0)$ is a simple curve, piecewise smooth such that $\left|G\left(\varphi\left(t, x_{0}, y_{0}\right)\right)\right| \leq \delta$, for $t \in\left[t_{1}, t_{2}\right]$ and for any $\left(x_{0}, y_{0}\right) \in \mathcal{A}$.

Consider the planar singular perturbation problem

$$
x^{\prime}=\alpha(x, y, \varepsilon), \quad y^{\prime}=\varepsilon \beta(x, y, \varepsilon) .
$$

If $\varepsilon=0$ then the fast flow is horizontal. The slow flow on the center manifold $\alpha(x, y, 0)=0$ is determined by the equation $y^{\prime}=\beta(x, y, 0)$.

Lemma 2. Consider the singular perturbation problem (8). Suppose that $\alpha, \beta: \mathbb{R}^{2} \rightarrow \mathbb{R}$ are $C^{r}$ functions satisfying that $\frac{\partial \alpha}{\partial x}(x, y, 0)<0, \frac{\partial \alpha}{\partial y}(x, y, 0) \neq$ 0 and $\beta(x, y, 0) \neq 0$ for any $(x, y) \in \mathbb{R}^{2}$ satisfying $\alpha(x, y, 0)=0$. Given $\left(x_{0}, y_{0}\right) \in \mathbb{R}^{2}$ and $\delta>0$ there exist $\varepsilon_{0}>0,0<t_{1}<t_{2}$ and $\left(x_{0}, y_{0}\right) \in \mathcal{A} \subseteq \mathbb{R}^{2}$ such that for $0<\varepsilon<\varepsilon_{0}$ system (8) is $\delta$-synchronized for $0<\varepsilon<\varepsilon_{0}$, $t \in\left[t_{1}, t_{2}\right]$ and $(x, y) \in \mathcal{A} \subseteq \mathbb{R}^{2}$.

Proof. Since $\frac{\partial \alpha}{\partial x}(x, y, 0)<0, \frac{\partial \alpha}{\partial y}(x, y, 0) \neq 0$ it follows that the slow manifold is a graphic of a monotone function. Besides, this graphic is composed by equilibrium points of the fast system which are attracting in the $x$-direction. Moreover $\beta(x, y, 0) \neq 0$ on the slow manifold implies that the reduced flow has no equilibrium points. Denote $A_{\delta / 2}$ a tubular neighborhood of the slow manifold with width $\delta / 2$. Given $\left(x_{0}, y_{0}\right) \in \mathbb{R}^{2}$ we have that $\varphi^{0}\left(x_{0}, y_{0}, t_{0}\right) \in$ $A_{\delta / 2}$ for some $t_{0}>0$. The continuity of the flow implies the existence of $\varepsilon_{0}, t_{1}, t_{2}$ and $\mathcal{A}$ satisfying the statement of the lemma. 


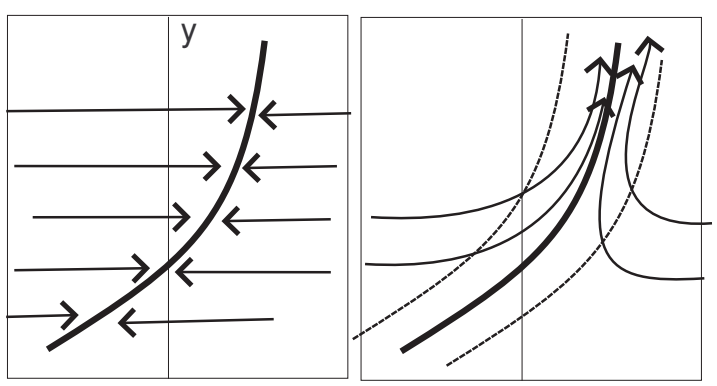

Figure 8. Fast and slow flow of a singular perturbation problem satisfying the hypothesis of Lemma 2 and the synchronized vector field.

Theorem 3. Suppose that $\Sigma^{S}=\Sigma$. Given $\delta>0$ there exist $\varepsilon_{0}>0,0<$ $t_{1}<t_{2}$ and $\left(x_{0}, y_{0}\right) \in \mathcal{A} \subseteq \mathbb{R}^{2}$ such that for $0<\varepsilon<\varepsilon_{0}$ the regularized vector field $X_{\varepsilon}$ is $\delta$-synchronized for $0<\varepsilon<\varepsilon_{0}, t \in\left[t_{1}, t_{2}\right]$ and $(x, y) \in \mathcal{A} \subseteq \mathbb{R}^{2}$.

Proof. We can choose a coordinate system such that the discontinuous set is defined by the function $H(x, y)=x$. Denote $X^{+}=\left(f^{+}, g^{+}\right)$and $X^{-}=$ $\left(f^{-}, g^{-}\right)$. Next we consider the blow up $x=r \cos \theta, \varepsilon=r \sin \theta$. Thus, the trajectories of the regularized vector fields, in polar coordinates, satisfy the differential system

$$
r \theta^{\prime}=\alpha(r, \theta, y), \quad y^{\prime}=\beta(r, \theta, y)
$$

with $\alpha, \beta$ given by $\alpha(r, \theta, y)=-\sin \theta\left(\frac{f_{+}+f_{-}}{2}+\varphi(\cot \theta) \frac{f_{+}-f_{-}}{2}\right)$, and $\beta(r, \theta, y)=r\left(\frac{g_{+}+g_{-}}{2}+\varphi(\cot \theta) \frac{g_{+}-g_{-}}{2}\right)$. According to the proof of Theorem A, the slow manifold $\alpha(0, \theta, y)=0$ is homeomorphic to the sliding region $\Sigma^{\mathcal{S}}$. In this coordinate system we have $\Sigma^{\mathcal{S}}=\Sigma=\{(0, y) ; y \in \mathbb{R}\}$. The singular perturbation problem satisfies the hypothesis of Lemma 2 . It means that given $\delta>0$ there exists $r_{0}>0$ such that for $0<r<r_{0}$ the above system is $\delta$-synchronized. The result follows after going back through the blow ups.

\section{Acknowledgments}

The first author is partially supported by the MICIIN/FEDER grant MTM2008-03437, the Generalitat de Catalunya grant 2009SGR-410 and ICREA Academia. The second author and the third author were partially supported by a CNPq and FAPESP. All the authors are supported by the Int. Coop. Proj. CAPES/MECD-TQED II and PHB-2009-0025. 


\section{REFERENCES}

[1] Alberto, L.F.C., Bretas, N.G. and Rodrigues, H. (2001). Uniforme Invariance Principle and Synchronizations. Robustness with Respect to Parameter Variation, J. Diff. Equations 169, 228-254.

[2] Broucke, M. et al (2001). Structural stability of piecewise smooths systems, Computational \& Applied Mathematics 20, 51-90.

[3] Buzzi, C., Silva, P.R. and Teixeira, M.A. (2006). A Singular approach to discontinuous vector fields on the plane, J. Diff. Equations 231, 633-655.

[4] Chow, S.N. and Liu, W. (1997). Synchronization, stability and normal hyperbolicity, Resenhas IME-USP 3-1, 139-158.

[5] di Bernardo, M. et al(2008). Bifurcations in non-smooth dynamical systems, SIAM Review 50, 629-701.

[6] Dumortier, F. Llibre, J. and Artés, J.C. (2006) Qualitative Theory of Planar Differential Systems, Universitex, Springer-Verlag.

[7] Dumortier, F. and Roussarie, R. (1996). Canard cycles and center manifolds, Memoirs Amer. Mat. Soc. 121.

[8] Fenichel, N. (1979). Geometric singular perturbation theory for ordinary differential equations, J. Diff. Equations 31, 53-98.

[9] Filippov, A. F. (1988). Differential Equations with Discontinuous Righthand Sides, Mathematics and its Applications ( Soviet Series), Kluwer Academic Publishers, Dordrecht.

[10] Llibre, J., Silva, P.R. and Teixeira, M.A. (2007). Regularization of Discontinuous Vector Fields via Singular Perturbation, J. Dynam. Differential Equation 19, 309331.

[11] Llibre, J., Silva, P.R. and Teixeira, M.A. (2008). Sliding vector fields via slow fast systems, Bulletin of the Belgian Mathematical Society Simon Stevin 15, 851-869.

[12] Llibre, J., Silva, P.R. and Teixeira, M.A. (2009). Study of Singularities in non smooth dynamical systems via Singular Perturbation, SIAM Journal on Applied Dynamical Systems 8, 508-526.

[13] Llibre, J., and Teixeira, M.A. (2010). On the stable limit cycle of a weight-driven pendulum clock, Eur. J. Phys. 31, 1249-1254.

[14] Sotomayor, J. and Teixeira, M.A. (1996). Regularization of Discontinuous Vector Fields, International Conference on Differential Equations, Lisboa, Equadiff 95, 207223.

${ }^{1}$ Departament de Matemàtiques, Universitat Autònoma de Barcelona, 08193 Bellaterra, Barcelona, Catalonia, Spain.

2 Departamento de Matemática - ibilce-Unesp, Rua C. Colombo, 2265, CEP 15054-000 S. J. Rio Preto, SÃo Paulo, Brazil

3 IMECC-UNICAMP, CEP 13081-970, Campinas, SÃo Paulo, Brazil

E-mail address: jllibre@mat.uab.cat

E-mail address: prs@ibilce.unesp.br

E-mail address: teixeira@ime.unicamp.br 